\title{
Unified Out-of-Band Emission Reduction with Linear Complexity for OFDM
}

\author{
Xiaojing Huang, Jian A. Zhang, and Y. Jay Guo \\ CSIRO Digital Productivity and Services, Sydney, Australia \\ Emails: \{Xiaojing.Huang, Andrew.Zhang, Jay.Guo\} @csiro.au
}

\begin{abstract}
This paper proposes a unified out-of-band emission (OOBE) reduction framework with linear complexity for orthogonal frequency-division multiplexing (OFDM) systems. Unlike conventional spectral precoding approaches which use orthogonal precoding matrixes, this framework composes cancellation signals from the linear combinations of data symbols and minimizes the average OOBE power with a general least-squares solution. A joint frequency domain cancellation subcarrier and data domain cancellation symbol allocation scheme is also proposed for discrete Fourier transform precoded OFDM, by which the overall signal processing complexity of the OFDM transceiver is further reduced without impact on other system performance. The advantages of the proposed scheme is verified both analytically and by simulation as compared with some well-known low-complexity OOBE reduction schemes.
\end{abstract}

Index Terms-Cognitive radio, OFDM, out-of-band emission, and peak-to-average power ratio.

\section{INTRODUCTION}

Orthogonal frequency-division multiplexing (OFDM) has been widely used in various communications systems, such as the wireless local area networks (WLANs), the 4th generation mobile systems [1], and other high speed microwave systems [2]. It is also very suitable for multiband cognitive radio systems [3] due to its flexibility for subcarrier allocation. A frequency band can be dynamically selected or de-selected by turning on or off the subcarriers falling in the band, such that the spectrum can be efficiently shared between licensed users (primary users) and unlicensed users (secondary users). However, OFDM also has some drawbacks. One of them is the significant out-of-band emission (OOBE) due to the slow sidelobe roll-off of the subcarriers. Without effective OOBE reduction, interference would occur in adjacent bands and therefore degrade the system performance.

There are a number of existing techniques developed for OFDM OOBE reduction. The first straightforward technique is to use a spectral shaping filter. However, a digital implementation of this filter would increase considerably the signal processing complexity, and an analogue implementation would be less cost effective or flexible for dynamic spectrum allocation.

The second technique is to introduce guard band between two adjacent transmission bands, i.e., nulling the subcarriers located on the edges of a given frequency band. Unfortunately, this will sacrifice spectral efficiency and may not be able to provide sufficient protection without using a significantly large number of null subcarriers.
The third technique is to perform windowing to the time domain OFDM signal. This would require an extended OFDM symbol with extra signal power and cause inter-symbol interference. Guard bands may also be needed together with windowing to ensure satisfactory OOBE reduction.

The fourth technique is to use dedicated subcarriers, called cancellation carriers, to cancel OOBE [4,6]. To achieve sufficient OOBE reduction, significant power should be allocated on the cancellation carriers, resulting in reduced power efficiency. Inserting cancellation carriers may also increase peakto-average power ratio (PAPR) of the OFDM signal.

The fifth technique is the spectral precoding method which has attracted significant research interests in recent years [5,8-10]. The general approach for spectral precoding is to use a data-independent matrix to precode the data symbols in the frequency domain to reduce OOBE. It provides more effective and dynamic OOBE reduction at the cost of higher computational complexity as it generally requires matrix multiplications at both transmitter and receiver. A lowcomplexity sidelobe suppression with orthogonal projection (SSOP) scheme has been proposed in [9]. However, the biterror-rate (BER) performance is slightly degraded due to the noise enhancement for data symbol recovery at receiver.

In this paper, a general linear complexity OOBE reduction framework for OFDM is proposed. It unifies a few well known spectral precoding schemes and can be flexibly configured to generate new schemes according to specific requirements on the complexity and performance for OOBE reduction. We also propose to use explicit frequency domain cancellation subcarriers (FDCSs) and data domain cancellation symbols (DDCSs) for OOBE reduction in precoded OFDM, which achieves significant OOBE reduction without increasing signal PAPR or degrading BER performance. The complexity required for OOBE reduction, which is proportional to the number of the total in-band subcarriers, is only incurred at transmitter without extra signal processing for data symbol recovery at receiver.

The rest of this paper is organized as follows. In Section II, the OFDM signal and its power spectral density are formulated. The general OOBE cancellation framework is then presented in Section III. In Section IV, the proposed framework is applied to precoded OFDM, in which explicit FDCSs and/or DDCSs are used. Section V provides performance comparison among various OOBE reduction schemes. Finally, conclusions are drawn in Section VI. 


\section{OFDM SIGNAL AND OOBE}

OFDM is a multicarrier transmission technique by which information data symbols are modulated on frequency domain subcarriers. Time domain OFDM symbols are obtained by performing inverse fast Fourier transform (IFFT) on frequency domain subcarriers.

Let $X(k), 0 \leq k \leq M-1$, denote the data symbols after constellation mapping on $M$ subcarriers and assume that the IFFT size is $N$, satisfying $N>M$. The discrete-time OFDM symbol can be expressed as

$$
x(n)=\frac{1}{\sqrt{N}} \sum_{k=0}^{M-1} X(k) e^{j \frac{2 \pi}{N} k n} g(n)
$$

where $g(n)=\left.g(t)\right|_{t=n T_{s}}, g(t)$ is a continuous-time window function, and $T_{s}$ is the sampling period. $g(t)$ can be a rectangular function with duration equal to $N T_{s}$ (corresponding to an OFDM symbol with zero-padding) or longer than $N T_{s}$ (corresponding to an OFDM symbol with cyclic prefix. The frequency domain representation of $x(n)$ can be expressed as

$$
\begin{aligned}
\tilde{X}(f) & =\sum_{n=-\infty}^{+\infty} x(n) e^{-j 2 \pi f n T_{s}} \\
& =\frac{1}{\sqrt{N}} \sum_{n=-\infty}^{+\infty} \sum_{k=0}^{M-1} X(k) e^{j \frac{2 \pi}{N} k n} g(n) e^{-j 2 \pi f n T_{s}} \\
& =\frac{1}{\sqrt{N}} \sum_{k=0}^{M-1} X(k) \tilde{G}\left(f-\frac{k}{N T_{s}}\right)
\end{aligned}
$$

where

$$
\tilde{G}(f)=\sum_{n=-\infty}^{+\infty} g(n) e^{-j 2 \pi f n T_{s}}=\frac{1}{T_{s}} \sum_{k=-\infty}^{+\infty} G\left(f-\frac{k}{T_{s}}\right)
$$

and $G(f)=\int_{-\infty}^{+\infty} g(t) e^{-j 2 \pi f t} d t$ are the frequency domain representations of $g(n)$ and $g(t)$ respectively. Note that $\tilde{X}(f)$ and $\tilde{G}(f)$ are periodic functions with period $\frac{1}{T_{s}}$.

The power spectral density (PSD) of $x(n)$ is

$$
\begin{aligned}
\tilde{S}(f)= & E\left\{|\tilde{X}(f)|^{2}\right\}=\frac{1}{N} \sum_{k=0}^{M-1} \sum_{k^{\prime}=0}^{M-1} E\left\{X(k) X^{*}\left(k^{\prime}\right)\right\} \\
& \cdot \tilde{G}\left(f-\frac{k}{N T_{s}}\right) \tilde{G}^{*}\left(f-\frac{k^{\prime}}{N T_{s}}\right)
\end{aligned}
$$

where $E\{\cdot\}$ denotes the ensemble averaging and $E\left\{X(k) X^{*}\left(k^{\prime}\right)\right\}$ is the correlation among the data symbols. Suppose that a pulse shaping filter with impulse response $p(t)$ is used to convert the discrete-time signal $x(n)$ to a continuous-time one. The final transmitted analogue OFDM signal will have the PSD

$$
S(f)=|P(f)|^{2} \tilde{S}(f)
$$

where $P(f)$ is the Fourier transform of $p(t)$, i.e., the frequency response of the pulse shaping filter.
Apparently, if $X(k)$ for $0 \leq k \leq M-1$ are independent with unit variance, the PSD of the OFDM signal becomes

$$
S(f)=|P(f)|^{2} \sum_{k=0}^{M-1}\left|\tilde{G}\left(f-\frac{k}{N T_{s}}\right)\right|^{2} .
$$

If the pulse shaping filter is not sharp enough, the transmitted power outside the signal spectrum $0 \leq f \leq \frac{M}{N T_{s}}$, referred to as OOBE, will be significant due to the slow decay of the sidelobes of $G(f)$, which is in the order of $\frac{1}{|f|}$.

\section{General OOBE CANCELlation Framework}

From (6) we see that designing a proper pulse shaping filter and/or selecting a suitable window function would be able to reduce OOBE. However, there are a lot of disadvantages as mentioned in the Introduction section. From (4) we see that changing the data symbol correlation through conventional spectral precoding could also reduce OOBE, but it would increase the implementation complexity.

We now propose a general linear precoding framework for low-complexity OOBE reduction. For simplicity, we denote the original data symbols as an $M \times 1$ column vector $\mathbf{X}=\left(\begin{array}{llll}X(0) & X(1) & \ldots & X(M-1)\end{array}\right)^{T}$, where $(\cdot)^{T}$ denotes matrix transposition, and the data symbols after OOBE cancellation as an $M \times 1$ column vector $\mathbf{Y}=$ $\left(\begin{array}{llll}Y(0) & Y(1) & \ldots & Y(M-1)\end{array}\right)^{T}$. The proposed method is derived as follows.

First, we define an attenuation matrix $\mathbf{A}$ of dimension $Q \times M$, where $Q$ is the number of normalized frequencies (normalized by subcarrier spacing $\left.\frac{1}{N T_{s}}\right) \omega_{q}, q=0,1, \cdots, Q-1$, outside the transmission frequency band, called cancellation points. The element at the $q$ th row and the $m$ th column in $\mathbf{A}$ is the attenuation factor by which the data symbol $X(\mathrm{~m})$ at subcarrier $m$ produces an OOBE at the cancellation point $\omega_{q}$, i.e., $\frac{1}{\left|\omega_{q}-m\right|}$. With this definition, $\mathbf{A X}$ will be the total OOBE caused by signal vector $\mathbf{X}$ at all specified cancellation points.

Second, we define a cancellation matrix $\mathbf{C}$ of dimension $L \times M$, where $L$ is the number of cancellation values used to cancel the OOBE caused by $\mathbf{X}$. The $L$ cancellation values are calculated from $\mathbf{X}$ as $\mathbf{C X}$, i.e., the $L$ linear combinations of data symbols. We will show how to determine the cancellation matrix $\mathbf{C}$ at the final step.

Third, we define a distribution matrix $\mathbf{D}$ of dimension $M \times L$, which distributes the cancellation values $\mathbf{C X}$ onto all subcarriers in the transmission frequency band to produce cancellation signal DCX. Then, we have

$$
\mathbf{Y}=\mathbf{X}-\mathbf{D C X}
$$

which are the data symbols after OOBE cancellation and used to generate time domain OFDM symbol via IFFT. Multiplying $\mathbf{Y}$ by the attenuation matrix $\mathbf{A}, \mathbf{A Y}$ represents the residual OOBE at the specified cancellation points $\omega_{q}$ for $q=0,1, \cdots, Q-1$. 
Finally, we determine the cancellation matrix $\mathbf{C}$ by minimizing the residual OOBE power

$$
\begin{aligned}
E & \left\{\|\mathbf{A Y}\|^{2}\right\}=E\left\{\|(\mathbf{A}-\mathbf{A D C}) \mathbf{X}\|^{2}\right\} \\
& =E\left\{\operatorname{tr}\left\{(\mathbf{A}-\mathbf{A D C}) \mathbf{X} \mathbf{X}^{H}(\mathbf{A}-\mathbf{A D C})^{H}\right\}\right\} \\
& =\operatorname{tr}\left\{(\mathbf{A}-\mathbf{A D C}) E\left\{\mathbf{X X}^{H}\right\}(\mathbf{A}-\mathbf{A D C})^{H}\right\} \\
& =\|\mathbf{A}-\mathbf{A D C}\|^{2}
\end{aligned}
$$

where $\|\cdot\|^{2}$ denotes the Frobenius norm of a matrix, $(\cdot)^{H}$ the matrix transposition and conjugation, and $\operatorname{tr}\{\cdot\}$ the trace of a matrix. The least-squares solution of the unconstrained minimization problem $\min _{\mathbf{C}}\|\mathbf{A}-\mathbf{A D C}\|^{2}$ is found to be

$$
\mathbf{C}=(\mathbf{A D})^{+} \mathbf{A}
$$

where $(\cdot)^{+}$denotes the pseudo-inverse of a matrix. Under different $Q$ and $L$ conditions, $(\mathbf{A D})^{+}$can be calculated by

$$
(\mathbf{A D})^{+}=\left\{\begin{array}{ll}
(\mathbf{A D})^{-1}, & Q=L \\
(\mathbf{A D})^{H}\left(\mathbf{A D}(\mathbf{A D})^{H}\right)^{-1}, & Q<L \\
\left((\mathbf{A D})^{H} \mathbf{A D}\right)^{-1}(\mathbf{A D})^{H}, Q>L
\end{array} .\right.
$$

The above described framework generalizes some existing OOBE reduction techniques, such as the unconstrained cancellation carrier (CC) method [4], the self-cancellation for SC-FDMA [7], and the SSOP [9]. These techniques can be recast as a general linear operation and linked to the proposed framework by adequately choosing the $\mathbf{D}$ matrix. For example, when $\mathbf{D}$ is selected as $\left(\begin{array}{cccc}1 & 0 & \cdots & 0 \\ 0 & \cdots & 0 & 1\end{array}\right)^{T}$, the cancellation carrier method is obtained with a frequency domain cancellation subcarrier located on each side of the transmission band; when $\mathbf{D}$ is selected as $\left(\begin{array}{cccc}1 & 1 & \cdots & 1\end{array}\right)^{T}$, the self-cancellation method for SC-FDMA is obtained, which equivalently uses the direct current component of the discrete Fourier transform (DFT) precoded data symbols for OOBE reduction; and when $\mathbf{D}$ is selected as $\mathbf{A}^{T}$, the SSOP method is obtained.

From (7), $\mathbf{Y}$ can be expressed as $\mathbf{Y}=\left(\mathbf{I}_{M}-\mathbf{D C}\right) \mathbf{X}$ where $\mathbf{I}_{M}$ is the identity matrix of order $M$. So, the proposed framework is also a kind of spectral precoding. However, the precoding matrix is not an orthogonal matrix, but structured in such a way that low-complexity can be achieved.

\section{OOBE REDUCTION FOR DFT PRECODED OFDM}

Precoded OFDM is a variation of conventional OFDM, where the original data symbols are pre-processed before IFFT. Mathematically, precoding is a matrix multiplication with the data symbol vector. Despite the additional complexity, this operation will enhance some of the OFDM signal's properties such as reducing PAPR, improving frequency diversity, as well as reducing OOBE. For clarity, we refer to the signal domain before precoding as the data domain, whereas the signal domains before and after IFFT as the frequency domain

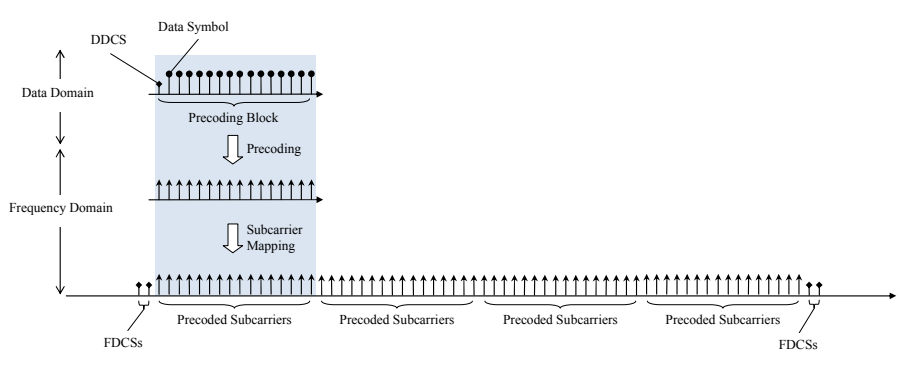

Fig. 1. Example of DDCS and FDCS allocation.

and the time domain respectively. In this section, we demonstrate how to allocate explicit cancellation subcarriers in the frequency domain (i.e., FDCSs) and cancellation symbols in the data domain (i.e., DDCSs) for efficient OOBE reduction in precoded OFDM system (i.e., $\mathbf{X}$ is precoded) and at the same time preserve some of the precoded OFDM's advantages.

The precoding matrix can be a single matrix, or, consist of several sub-matrices. Each matrix can be orthogonal or non-orthogonal, but shall be invertible such that the original data symbols can be recovered at the receiver. Without loss of generality, we consider the case where each sub-matrix is a DFT matrix. Using a DFT matrix can reduce implementation complexity thanks to FFT and also reduce the PAPR of the transmitted OFDM signal.

To achieve effective OOBE reduction, DDCS and FDCS are allocated before and after DFT precoding respectively, as illustrated in Fig. 1. In this example, four DDCSs and four FDCSs in the considered frequency band are allocated. In the data domain, data symbols are grouped into four precoding blocks (only one precoding block is shown in the shaded area). In each precoding block, one DDCS is placed at the first symbol position. In the frequency domain, two FDCSs are placed on each edge of the transmission band.

With the above explicit DDCS and FDCS allocation, the distribution matrix $\mathbf{D}$ is then designed as follows.

If the $m$ th subcarrier is an FDCS and it takes on the $l$ th cancellation value in $\mathbf{C X}, l=0,1, \cdots, L-1$, the element at the $m$ th row and the $l$ th column in $\mathbf{D}$ is set to 1 and the remaining elements in the row are set to 0s. Otherwise, all the elements in the row are set to 0s, unless the arrangements for DDCSs over-write them. For example, for an FDCS located at subcarrier 0 , the corresponding column in $\mathbf{D}$ is $\left(\begin{array}{llll}1 & 0 & \ldots & 0\end{array}\right)^{T}$. This ensures that a cancellation value for an FDCS will not be distributed to other subcarriers.

When a precoded block of size $K$ is allocated to subcarriers $m$ to $m+K-1$ and a DDCS in the corresponding precoding block takes on the $k$ th $(k \neq l)$ cancellation value, the $m$ th to $(m+K-1)$ th elements in the $k$ th column in $\mathbf{D}$ are set to 1s. For example, for a DDCS allocated in the precoding block which produces $K$ precoded subcarriers with indexes 2 to $2+K-1$, the corresponding column in $\mathbf{D}$

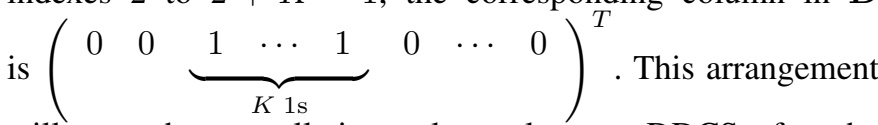
will map the cancellation value only to a DDCS after the 
inverse DFT and hence will not cause interference to other data symbols.

One cancellation value will be distributed to one, and only one, FDCS or DDCS. This ensures that $\mathbf{D}$ will always be a full column-rank matrix.

From the above distribution matrix construction process, we see that $L$, the number of columns of $\mathbf{D}$, equals to the total number of FDCSs and DDCSs, i.e., the precoding redundancy[8].

Compared with existing OOBE reduction techniques, the proposed framework using explicit FDCSs and DDCSs has the following advantages. First, it has very low complexity. According to (7), the number of multiplication operations is only $L M$ for calculating $\mathbf{C X}$ at transmitter where $\mathbf{C}$ is a real-valued matrix, whereas conventional spectral precoding would require $M^{2}$ complex multiplications. The data symbols are free from interference after DFT de-precoding at the receiver, therefore, no extra signal processing is required for data symbol recovery. Second, despite the slight increase of transmitted signal power due to the insertion of FDCSs and DDCSs, the data symbols are not distorted by any FDCS or DDCS, so that there will be no BER performance degradation under the same effective signal power carried on data symbols. Third, as will be demonstrated later, jointly using FDCSs and DDCSs for OOBE reduction also produces lower PAPR for the transmitted OFDM signal.

\section{PERFormance COMPARISON VIA ANALYSIS AND SIMULATION}

In this section, we compare the OOBE reduction performance among different methods under the unified framework. For convenience, we only consider the case $Q>L$ and select the cancellation points as all out-of-band subcarriers away from the left and right sides of the transmission band with offset $\omega_{L}$ and $\omega_{R}$ (called left and right cancellation distances) respectively.

Considering that the $\mathbf{D}$ and $\mathbf{A}$ matrices are real-valued, the C matrix can be simplified from (9) and (10) as

$$
\begin{aligned}
\mathbf{C} & =\left((\mathbf{A D})^{H} \mathbf{A D}\right)^{-1}(\mathbf{A D})^{H} \mathbf{A} \\
& =\left(\mathbf{D}^{T} \mathbf{A}^{T} \mathbf{A} \mathbf{D}\right)^{-1} \mathbf{D}^{T} \mathbf{A}^{T} \mathbf{A} \\
& =\left(\mathbf{D}^{T} \mathbf{\Phi} \mathbf{D}\right)^{-1} \mathbf{D}^{T} \mathbf{\Phi}
\end{aligned}
$$

where $\boldsymbol{\Phi}=\mathbf{A}^{T} \mathbf{A}$ is referred to as the kernel matrix of dimension $M \times M$. An element in $\boldsymbol{\Phi}$ at the $i$ th row and the $j$ th column is defined as the kernel function $\varphi(i, j)$, which can be evaluated using a closed-form approximation as

$$
\begin{aligned}
\varphi(i, j) & =\sum_{\omega=\omega_{L}}^{\infty} \frac{1}{(\omega+i)(\omega+j)} \\
& +\sum_{\omega=\omega_{R}}^{\infty} \frac{1}{(\omega+M-1-i)(\omega+M-1-j)} \\
& \approx\left\{\begin{array}{ll}
\frac{1}{i-j} \ln \frac{\left(\omega_{L}+i\right)\left(\omega_{R}+M-1-j\right)}{\left(\omega_{L}+j\right)\left(\omega_{R}+M-1-i\right)}, & i \neq j \\
\frac{1}{\omega_{L}+i}+\frac{1}{\omega_{R}+M-1-i}, & i=j
\end{array} .\right.
\end{aligned}
$$

The PSD of the OFDM signal after OOBE reduction can be obtained analytically as follows: For an OOBE reduction method with $\mathbf{D}$ matrix specified, the $\mathbf{C}$ matrix is calculated by (11) first. The PSD is then evaluated by (4) and (5) with $E\left\{X(k) X^{*}\left(k^{\prime}\right)\right\}$ replaced by $E\left\{Y(k) Y^{*}\left(k^{\prime}\right)\right\}$ which is the element at the $k$ th row and the $k^{\prime}$ th column of the correlation matrix

$$
\begin{aligned}
E\left\{\mathbf{Y} \mathbf{Y}^{H}\right\} & =\left(\mathbf{I}_{M}-\mathbf{D C}\right) E\left\{\mathbf{X X}^{H}\right\}\left(\mathbf{I}_{M}-\mathbf{D C}\right)^{H} \\
& =\left(\mathbf{I}_{M}-\mathbf{D C}\right) \widehat{\mathbf{I}}_{M}\left(\mathbf{I}_{M}-\mathbf{D C}\right)^{H}
\end{aligned}
$$

where $\widehat{\mathbf{I}}_{M}$ is a diagonal matrix with diagonal element being 1 corresponding to a precoded subcarrier and 0 corresponding to an FDCS. For example, if two FDCSs are allocated on each side of the frequency band, $\widehat{\mathbf{I}}_{M}=$ $\operatorname{diag}\left\{\begin{array}{llllllll}0 & 0 & 1 & 1 & \cdots & 1 & 0 & 0\end{array}\right\}$.

The OOBE reduction performance can be also evaluated by simulation. We consider a zero-padded OFDM system occupying a transmission bandwidth of $M=68$ subcarriers. Two subcarriers on each side of the band are reserved as FDCSs. The remaining 64 subcarriers are divided into 2 or 4 blocks of precoded subcarriers, each block having 32 or 16 subcarriers. For each precoding block of 32 or 16 data domain symbols, one symbol is reserved as DDCS. The IFFT size is selected as $N=512$. For simplicity, the pulse shaping filter $p(t)$ is omitted and the PSD is taken as one period of $\tilde{S}(f)$. The analytical and simulated results on the PSD performance are in perfect match. With simulation, the PAPRs of the transmitted OFDM signals, measured using the complimentary cumulative density function (CCDF), i.e., the probability of PAPR greater than a threshold $P A P R_{0}$, are also compared.

Fig. 2 and Fig. 3 show respectively the PSDs and PAPRs of the OFDM signals with and without OOBE reduction. The cancellation distances are set to $\omega_{L}=\omega_{R}=1$. The OOBE reduction methods include the cancellation carrier method (denoted as CC) with four FDCSs only, the self-cancellation method for SC-FDMA (denoted as SC-FDMA) corresponding to $L=1$ since only one DDCS is used, the SSOP method (denoted as SSOP) with 4 cancellation points selected at $-\frac{N}{2}$, $-1, M$ and $M-1+\frac{N}{2}$, and the proposed method using both FDCSs and DDCSs (denoted as FDCS+DDCS). Two cases for the FDCS+DDCS method are considered: one uses two FDCSs each located at each side the frequency band and two DDCSs each located in a precoding block of size 32 (i.e., $L=4$ ) and the other uses 4 FDCSs, 2 on each side of the frequency band, and 4 DDCSs each located in a precoding block of size 16 (i.e., $L=8$ ). We see that the $\mathrm{CC}$ method requires significantly higher power on cancellation subcarriers and also causes larger PAPR. SC-FDMA has the lowest PAPR due to the DFT precoding but the OOBE self-cancellation is not so effective since the precoding redundancy is only 1 . The SSOP method can achieve comparable OOBE reduction performance and it does not affect the PAPR. The major disadvantage is the BER performance degradation as analyzed in [9]. The proposed method using both FDCSs and DDCSs can achieve better OOBE reduction and also improve PAPR. 


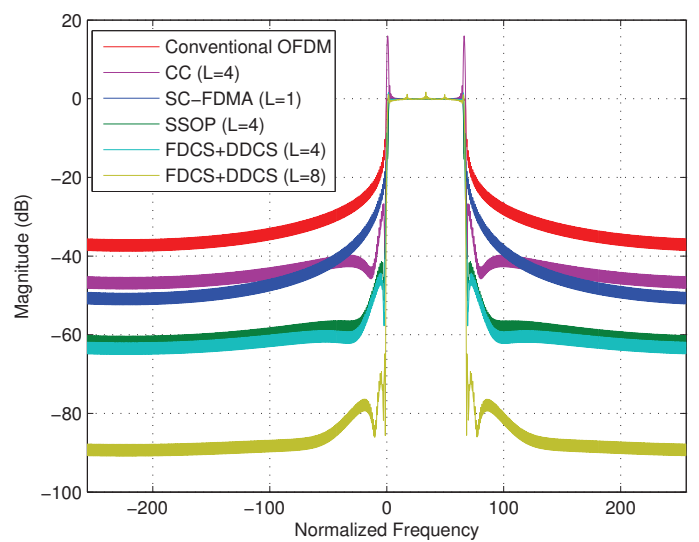

Fig. 2. PSDs of various OOBE reduction methods $\left(\omega_{L}=\omega_{R}=1\right)$.

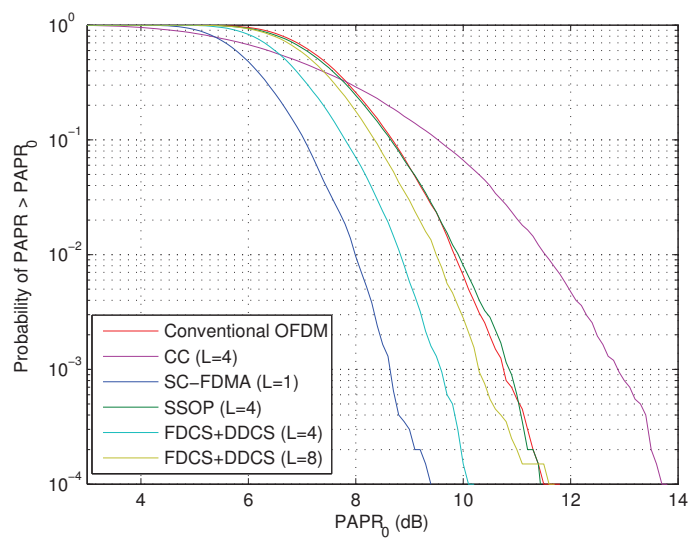

Fig. 3. PAPRs of various OOBE reduction methods $\left(\omega_{L}=\omega_{R}=1\right)$.

The cancellation distance also plays an important role in OOBE reduction. Generally speaking, if $\omega_{L}$ or $\omega_{R}$ is smaller, the OOBE will roll-off more quickly. With larger cancellation distance, the OOBE will roll-off more slowly but more OOBE reduction can be achieved. This is shown in Fig. 4 where PSDs of the precoded OFDM using the FDCS+DDCS approach with $L=4$ but various cancellation distances are displayed. The correspondng PAPR performance is also shown in Fig. 5. We see that the PAPR only increases slightly with larger cancellation distance but is still lower than that of the conventional OFDM.

\section{CONCLusions}

A unified linear complexity OOBE reduction framework for OFDM is presented in this paper. It has been shown that jointly using frequency domain cancellation subcarriers and data domain cancellation symbols for DFT precoded OFDM can achieve larger OOBE reduction than other existing methods with similar complexity. The PAPR performance is also improved at the same time. By properly designing the distribution matrix, the proposed framework provides great flexibility for an OFDM system to satisfy different performance requirements in different applications.

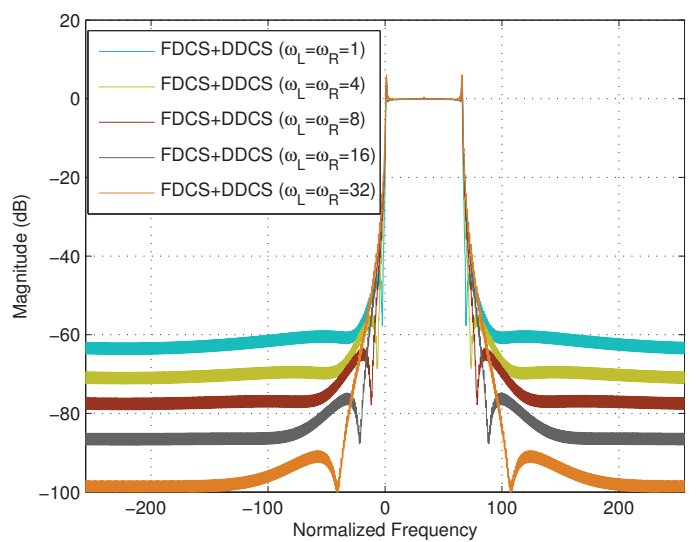

Fig. 4. PSDs of proposed method using both FDCSs and DDCSs $(L=4)$.

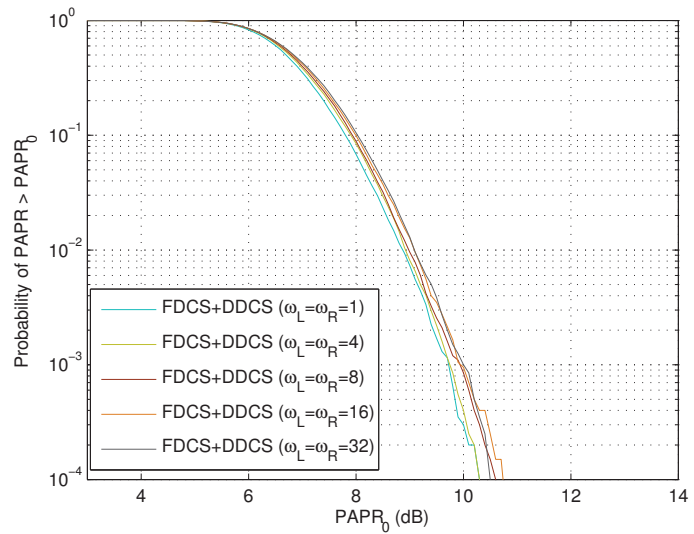

Fig. 5. PAPRs of proposed method using both FDCSs and DDCSs $(L=4)$.

\section{REFERENCES}

[1] S. Parkvall, et al., "Evolution of LTE toward IMT-Advanced," IEEE Communications Magazine, vol. 49, no. 2, pp. 84 - 91, February 2011.

[2] X. Huang, Y. J. Guo, J. Zhang, and V. Dyadyuk, "A multi-Gigabit microwave backhaul," IEEE Communications Magazine, vol. 50, no. 3, pp. 122 - 129, March 2012.

[3] S. Haykin, "Cognitive radio: Brain-empowered wireless communications," IEEE Journal of Selected Areas on Communications, vol. 23, no. 2, pp. $201-220$, February 2005.

[4] S. Brandes, I. Cosovic, and M. Schnell, "Reduction of out-of-band radiation in OFDM systems by insertion of cancellation carriers," IEEE Communications Letters, vol. 10, no. 6, pp. 420 - 422, June 2006.

[5] C.-D. Chung, "Spectral precoding for rectangularly pulsed OFDM," IEEE Transactions on Communications, vol. 56, no. 9, pp. 1498 - 1510, September 2008.

[6] D. Qu and Z. Wang, "Extended active interference cancellation for sidelobe suppression in cognitive radio OFDM systems with cyclic prefix," IEEE Transactions on Vehicular Technology, vol. 59, no.4, pp. 1689 - 1695, May 2010.

[7] M. Ma, X. Huang, and Y. J. Guo, "An interference self-cancellation technique for SC-FDMA systems," IEEE Communications Letters, vol. 14 , no. 6, pp. 512-514, June 2010.

[8] M. Ma, X. Huang, B. Jiao, and Y. J. Guo, "Optimal orthogonal precoding for spectral leakage suppression in DFT-based systems," IEEE Transactions on Comm., vol. 59, no. 3, pp. 844 - 853, March 2011.

[9] J. Zhang, X. Huang, T. Cantoni, and Y. J. Guo, "Sidelobe suppression with orthogonal projection for multicarrier systems," IEEE Transactions on Communications, vol. 60, no. 2, pp. 589 - 599, February 2012.

[10] X. Zhou, G. Y. Li, and G. Sun, "Multiuser spectral precoding for OFDMbased cognitive radio systems," IEEE Journal on Selected Areas in Communications, vol. 31, no. 3, pp. 345 - 352, March 2013. 